\title{
Communication \\ Ultra-Compact and Ultra-Broadband Polarization-Insensitive Mach-Zehnder Interferometer in Silicon-on-Insulator Platform for Quantum Internet Application
}

\author{
Jingjing Zhang ${ }^{1,2}$, Zhaojian Zhang ${ }^{2}$, Chao Ma ${ }^{1}$, Xuefeng Chen ${ }^{1}$, Liping Liu ${ }^{3}$, Wei Zhao ${ }^{2}$, Xiaoxian Song ${ }^{1}$, \\ Haiting Zhang ${ }^{1}$, Yu Yu ${ }^{4}$, Huan Chen ${ }^{2, *}$ and Junbo Yang ${ }^{2}$ \\ 1 Institute of Mirco/Nano Optoelectronic and Terahertz Technology, Jiang Su University, \\ Zhenjiang 210010, China; zhangjingjing13@nudt.edu.cn (J.Z.); mc@ujs.edu.cn (C.M.); \\ 2212003072@stmail.ujs.edu.cn (X.C.); songxiaoxian@ujs.edu.cn (X.S.); zhanghaiting@ujs.edu.cn (H.Z.) \\ 2 Department of Physics, National University of Defense Technology, Changsha 410073, China; \\ zhangzhaojian16@nudt.edu.cn (Z.Z.); zhaowei13@nudt.edu.cn (W.Z.); yangjunbo@nudt.edu.cn (J.Y.) \\ 3 State Key Laboratory of Pulsed Power Laser Technology, National University of Defense Technology, \\ Hefei 230037, China; liuliping10@nudt.edu.cn \\ 4 School of Electronic and Information Engineering, Hebei University of Technology, Tianjin 300401, China; \\ yuyu1990@hebut.edu.cn \\ * Correspondence: chenhuan11@nudt.edu.cn
}

Citation: Zhang, J.; Zhang, Z.; Ma, C.; Chen, X.; Liu, L.; Zhao, W.; Song, X.; Zhang, H.; Yu, Y.; Chen, H.; et al. Ultra-Compact and Ultra-Broadband Polarization-Insensitive Mach-Zehnder Interferometer in Silicon-on-Insulator Platform for Quantum Internet Application. Photonics 2021, 8, 455. https:// doi.org/10.3390/photonics 8100455

Received: 29 September 2021

Accepted: 13 October 2021

Published: 19 October 202

Publisher's Note: MDPI stays neutral with regard to jurisdictional claims in published maps and institutional affiliations.

Copyright: (c) 2021 by the authors. Licensee MDPI, Basel, Switzerland. This article is an open access article distributed under the terms and conditions of the Creative Commons Attribution (CC BY) license (https:// creativecommons.org/licenses/by/ $4.0 /)$

\begin{abstract}
Polarization dependence in integrated silicon photonics has a detrimental effect on the manipulation of quantum state with different polarizations in the quantum technology. Those limits have profound implications for further technological developments, especially in quantum photonic internet. Here, we propose a polarization-independent Mach-Zehnder interferometer (MZI) structure based on a $340 \mathrm{~nm}$-thick silicon-on-insulator (SOI) platform. The MZI facilitates low loss, broad operating bandwidth, and large tolerance of the fabrication imperfection. We achieve an excess loss of $<10 \%$ and an extinction radio of $>18 \mathrm{~dB}$ in the $100 \mathrm{~nm}$ bandwidth $(1500 \sim 1600 \mathrm{~nm})$ for both transverse electric (TE) and transverse magnetic (TM) modes. We numerically demonstrate an interference visibility of $99 \%$ and a polarization-independent loss (PDL) of $0.03 \mathrm{~dB}$ for both polarizations at $1550 \mathrm{~nm}$. Furthermore, by using the principle of phase compensation and self-image, we shorten the length of the waveguide taper by almost an order of magnitude with the transmission of $>95 \%$ for both TE and TM polarizations. Up to now, the proposed structure could significantly improve the integration and promote the development of monolithic integrated quantum internet.
\end{abstract}

Keywords: polarization insensitive; Mach-Zehnder interferometer; integrated optical

\section{Introduction}

Referring to the development of classical communication, the quantum information network will be the key technology in enabling long-distance quantum communication and assembling compact devices into a larger quantum computing cluster in the future [1]. Large-scale quantum computing and high-dimensional quantum communication suffer from the bulk optical element, bolted onto optical tables in free space and fiber systems [2]. Photonic integrated circuits (PICs) owing their low loss, compactivity and stability, have become a promising platform to realize photonic quantum interconnent. PICs have been demonstrated on different material platforms such as LiNO3, InP and SiN [3-6]. These waveguide materials with low refractive index contrasts are associated with large footprints and poor scalabilities.

Silicon-on-insulator (SOI) chips, which have high effective refractive index differences $(\Delta n \approx 2)$ between silicon and silicon dioxide, mean the silicon-based waveguide devices have strong confinement on light [7]. Compared with other materials, silicon-based devices are smaller in size and more suitable for system integration. Silicon PICs have been used to 
implement key components for quantum information processing, such as high-dimensional quantum entanglement, quantum walking, $\mathrm{CNOT}$ gates and factoring algorithms $[8,9]$. In quantum technology, the manipulation of a single photon in the time or frequency domain is the basic and key unit operation of quantum information coding/decoding, photon routing and quantum information processing. Most of these technologies require precise interferences, which is determined by the performance of Mach-Zehnder interferometer (MZI) structures.

Various silicon-based MZI structures have been demonstrated [10]. As an important part of the MZI, $3 \mathrm{~dB}$ power splitters have been widely studied with a variety of structures such as Y-junctions, directional couplers, grating beam splitters and multimode interference (MMI) couplers [11,12]. Among these structures, MMI-based power splitters have a small footprint, good tolerance to fabrication error and broad bandwidth. In 2012, D.B. et al. utilized an MMI-based MZI structure to realize quantum interference and the manipulation of entanglement in silicon wire waveguide quantum circuits [13]. The visibility of the MMI is $80 \%$ with a footprint of $2.8 \mu \mathrm{m} \times 27 \mu \mathrm{m}$ in the SOI platform and a silicon thickness of $220 \mathrm{~nm}$. In order to accurately control linear optical devices for large-scale quantum information processing and other applications [14-16], C. M. Wilkes et al. reported a high-extinction auto-configured Mach-Zehnder interferometer with a $60 \mathrm{~dB}$ range [17].

However, the MZI devices mentioned above based on SOI are generally polarization sensitive. Due to different effective refraction indexes for two orthogonal polarizations, it is difficult to fulfill the phase-matching condition on the same device [18-20], which will induce polarization dependence loss. Thus, as one of degrees of freedom for encoding in quantum information process, polarization dependence loss limits the applicability in communication systems. A possible solution requires quantum interconnections to enable scaling beyond the limit of monolithic integration, and towards multi-chip system, such as in Ref. [21], where the path-to-polarization conversion allows entangled quantum states to be coherently distributed only between silicon photonic integrated circuit and fiber path. Furthermore, the different polarization photons are coupled into a different silicon chip via a $2 \mathrm{D}$ grating coupler. The polarization dependent on the siliconintegrated circuit also makes it difficult to realize the point-to-point BB84 quantum key distribution (QKD) [22], let alone the BB84-based network. Therefore, it is meaningful to remove the polarization dependence of the silicon-based MZI. In 2018, D. Dai et al. proposed and experimentally demonstrated a polarization-insensitive $2 \times 2$ thermo-optic Mach-Zehnder switch by utilizing SOI nanophotonic waveguides with a $340 \mathrm{~nm}$-thick silicon core layer [23]. The extinction ratio of the structure was more than $20 \mathrm{~dB}$ in the C-band $(1530 \sim 1565 \mathrm{~nm})$ for both TE and TM polarizations. The MZI arms were designed with square SOI nanophotonic waveguide with a cross section of $340 \mathrm{~nm} \times 340 \mathrm{~nm}$, and could achieve polarization-insensitive phase shift. However, the polarization-dependent loss was as high as $2 \mathrm{~dB}$, and the $50 \%-50 \%$ power-splitting ratio was only in the wavelength range of $1550 \sim 1560 \mathrm{~nm}$, which is quite narrow for accurate photon manipulation. On the other hand, grating coupling was used to upload and download signals in the above references. The input field, which is coupled from fiber-adapted grating couplers, would mismatch with the field in single-mode waveguide. An adiabatic waveguide taper with a length of $>400 \mu \mathrm{m}$ can solve this problem, the corresponding large footprint degrades the compact of the photonic integrated circuit. Most of the grating couplers such as lens tapers [24], inverse design tapers [25] and interpolation formula tapers [26] are able to reduce the length to $\sim 40 \mu \mathrm{m}$; however, these structures always suffer from diffractive apertures which induce extra scattering loss, high crosstalk, uncertain phase variability, or complex fabrication processes, and only operate with TE or TM polarization states. Therefore, the footprint and the polarization dependence of input/output components should be considered in the design of MZI.

In this paper, we proposed a polarization-independent MZI, composed of a $1 \times 2$ beam splitter, phase shifter, and $2 \times 2$ beam splitters based on a $340 \mathrm{~nm}$ SOI platform, as shown in Figure 1. The $1 \times 2$ beam splitter utilizes with an MMI structure and has an ultra-short 
waveguide taper with a length of $20 \mu \mathrm{m}$ as the input port. For the experimental condition, the optical field can be injected from a grating coupler which is not shown in Figure 1. In this simulation, the light is directly injected in the taper with the fundamental mode. The input taper then narrows down to $2 \mu \mathrm{m}$ wide, being a multi-mode waveguide. The optical field in the multi-mode waveguide is in the first-order transverse electric $\left(\mathrm{TE}_{01} / \mathrm{TM}_{01}\right)$ mode. The multi-mode waveguide separates into two single-mode waveguides by the self-image principle. Then, after two phase-shift paths, the optical field interferes at the $2 \times 2$ beam splitter with a length of $11.6 \mu \mathrm{m}$ and width of $2 \mu \mathrm{m}$ [27]. In order to realize the same phase shift, the phase shifter can choose the graphene optical modulator, which can realize the polarization-insensitive modulator by careful design [28,29]. The transmissions of input and output waveguide mode converters are both higher than $95 \%$, with lengths of $24.1 \mu \mathrm{m}$ and $27.2 \mu \mathrm{m}$. The visibility of MZI reaches $99 \%$ and extinction ratio is $>20 \mathrm{~dB}$ with a wide wavelength bandwidth of $100 \mathrm{~nm}(1.5 \sim 1.6 \mu \mathrm{m})$ for both polarizations.

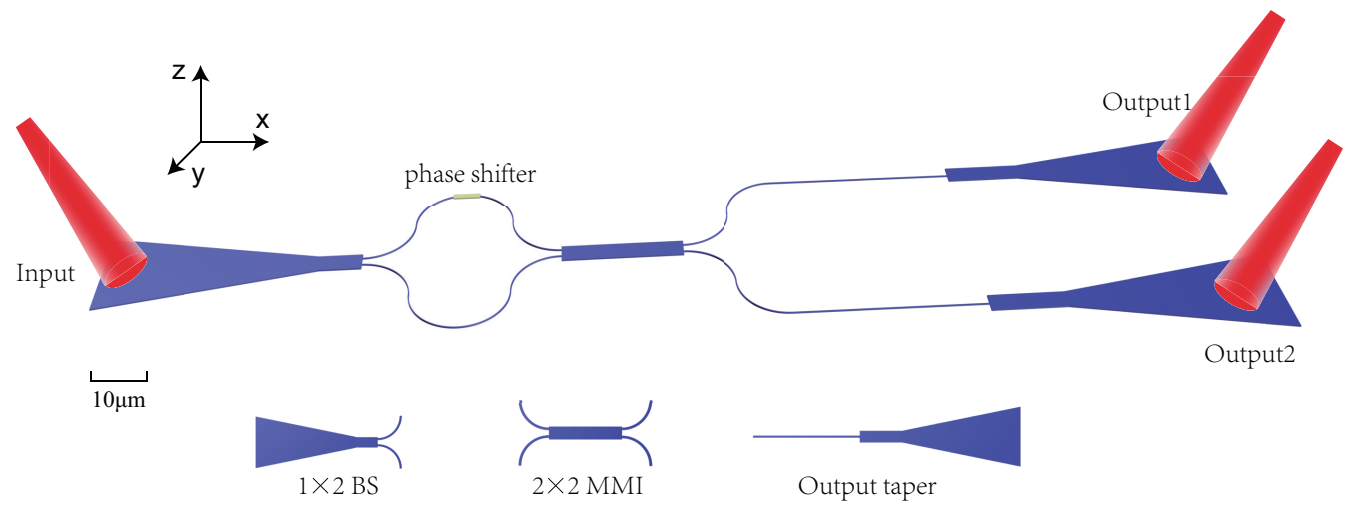

Figure 1. The schematic of the proposed Mach-Zhnder interferometer which mainly contains a $1 \times 2$ beam splitter, a phase shifter, a $2 \times 2$ beam splitters, and two output tapers. The length of $1 \times 2$ BS, output taper, and the footprint of $2 \times 2 \mathrm{MMI}$ are $24.1 \mu \mathrm{m}, 27.2 \mu \mathrm{m}$, and $2 \times 11.6 \mu \mathrm{m}^{2}$.

\section{Materials and Methods}

\subsection{The $1 \times 2$ Beam Splitter Design}

Self-imaging principle is usually used to analyze and design MMI-based devices [30]. The fundamental TE/TM mode is input from the single mode waveguide at the center to the multimode waveguide, which excites plenty of higher-order modes in the MMI region. By analyzing the field distribution of different waveguide modes in the multimode region of an MMI coupler, we can obtain the output field in different locations of the MMI coupler. The beat length $L_{\pi}$ of the fundamental mode and the first-order mode is given by $L_{\pi}=\pi / \Delta \beta$, where $\Delta \beta=\beta_{0}-\beta_{1}$, and $\beta_{0}\left(\beta_{1}\right)$ indicates the propagation constant of the $\mathrm{TE}_{0}\left(\mathrm{TE}_{1}\right)$ mode or $\mathrm{TM}_{0}\left(\mathrm{TM}_{1}\right)$ mode. When $\Delta \beta_{\mathrm{TE}}=\Delta \beta_{\mathrm{TM}}$, the MMI couplers are polarization independent. According to Ref. [23], the polarization-insensitive MMI coupler is designed at the width of $2 \mu \mathrm{m}$ based on $340 \mathrm{~nm}$-thickness SOI platform. Using the self-image theory, we obtain the primarily calculated values of the length, width, and gap (between output waveguides) of the multimode of the beam splitter, which are $4 \mu \mathrm{m}, 2 \mu \mathrm{m}$, and $0.5 \mu \mathrm{m}$, respectively. Using these values as initial values, we simulate the transmission with different multimode waveguide lengths, widths, gaps, and wavelengths at the optimized height of $340 \mathrm{~nm}$. The simulation is performed using 3D Lumerical FDTD solution [31]. The results are shown in Figure 2. We place monitors at the input port and output port, respectively, and finally normalize the energy to obtain the transmission.

Figure 2a shows the transmission versus multimode waveguide length at the width of $2 \mu \mathrm{m}$ and gap of $500 \mathrm{~nm}$ and the wavelength at the wavelength of $1.55 \mu \mathrm{m}$. At the length of $3.6 \mu \mathrm{m}$ to $4.7 \mu \mathrm{m}$, the beam splitter enables high transmission exceeding $40 \%$ for both polarizations. Meanwhile, the approximate equability transmission of TE polarization $(47.87 \%)$ and TM polarization $(47.82 \%)$ takes place at a length of $4.1 \mu \mathrm{m}$. The polarization dependence loss (PDL), which describes the polarization dependence of devices, reaches 0 . 
Therefore, the optimized length of beam splitters is $4.1 \mu \mathrm{m}$. The total transmission found by summing up the transmission at two channels is more than $95 \%$ for both polarizations. On the other hand, the optical field is normally coupled from lensed fibers or fiber-matched grating couplers to the input port of a taper with a $10 \mu \mathrm{m}$ wide and $20 \mu \mathrm{m}$ long multimode waveguide. The taper converts the multimode to the single mode with an output port of a $500 \mathrm{~nm}$-wide single mode waveguide. Thus, normally the length of the taper should be larger than $400 \mu \mathrm{m}$. Our structure has a length of only $24.1 \mu \mathrm{m}$, which would significantly improve the integration of the photonic integrated circuits.
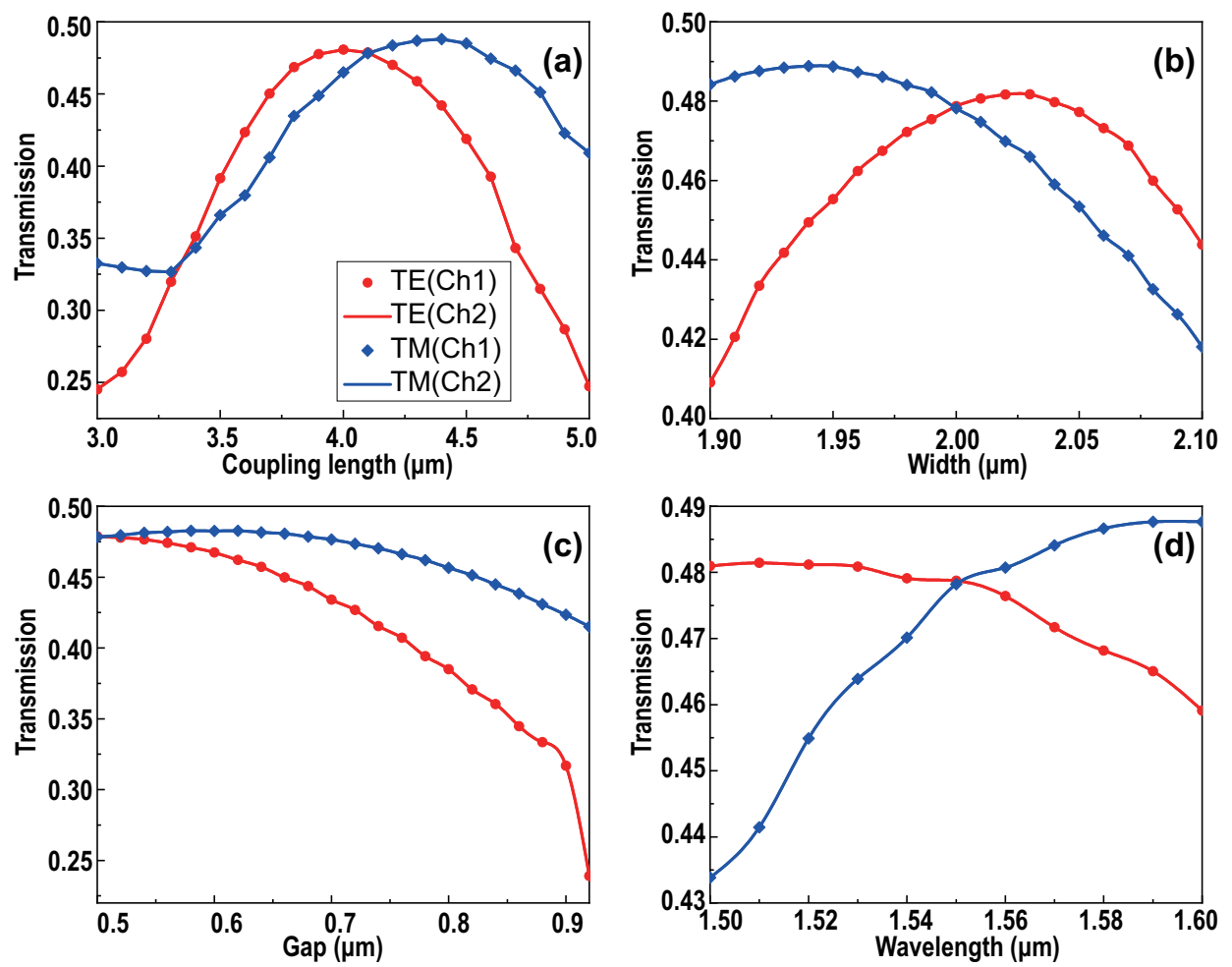

Figure 2. The transmission of the beam splitters versus (a) MMI length (b)MMI width (c) MMI gap width of channels (d) wavelength. TE polarization at SMW1: red circle; TE polarization at SMW2: red line; TM polarization at SMW1: blue diamond; TM polarization at SMW2: blue line.

The width of the MMI is a key parameter for the proposed beam splitter. Figure $2 b$ shows the transmission versus MMI width, with a length of $4.1 \mu \mathrm{m}$ and gap width of $500 \mathrm{~nm}$ at the wavelength of $1.55 \mu \mathrm{m}$. For the width from $1.9 \mu \mathrm{m}$ to $2.1 \mu \mathrm{m}$, the transmission is more than $40 \%$ for both polarizations. The structure is polarization independent at the width of $2.0 \mu \mathrm{m}$. The influence of the gap width is shown in Figure 2c. The length of the MMI is set to be $4.1 \mu \mathrm{m}$, the width of the MMI is set to be $2.0 \mu \mathrm{m}$, and the wavelength is $1.55 \mu \mathrm{m}$. In order to avoid energy coupling between the output waveguides, the width of the gap should be no less than $500 \mathrm{~nm}$. Thus, we vary the gap width from $500 \mathrm{~nm}$ to $900 \mathrm{~nm}$; the highest and equal transmission in the output of single mode waveguide 1 (SMW1) and 2 (SMW2) for both polarizations are obtained at the gap width of $500 \mathrm{~nm}$. Additionally, based on the optimized geometric dimension determined above, we obtain the transmission at different wavelengths shown in Figure 2. The transmission is more than $43 \%$ for both polarizations with a bandwidth of $100 \mathrm{~nm}$ (from $1.5 \mu \mathrm{m}$ to $1.6 \mu \mathrm{m}$ in wavelength range). The highest transmissions for the TE and TM mode are $48.14 \%$ and $48.77 \%$, taking place at $1.51 \mu \mathrm{m}$ and $1.6 \mu \mathrm{m}$, respectively, while the 0 PDL takes place at $1.55 \mu \mathrm{m}$. The transmission of the proposed structure is more than $86 \%$ for the $1 \mathrm{~dB}$ bandwidth $(100 \mathrm{~nm})$.

The energy distributions for both polarizations of the $1 \times 2$ beam splitter are shown in Figure 3. The incident fields for both TE and TM polarizations are converted and split into two single-mode waveguides. 

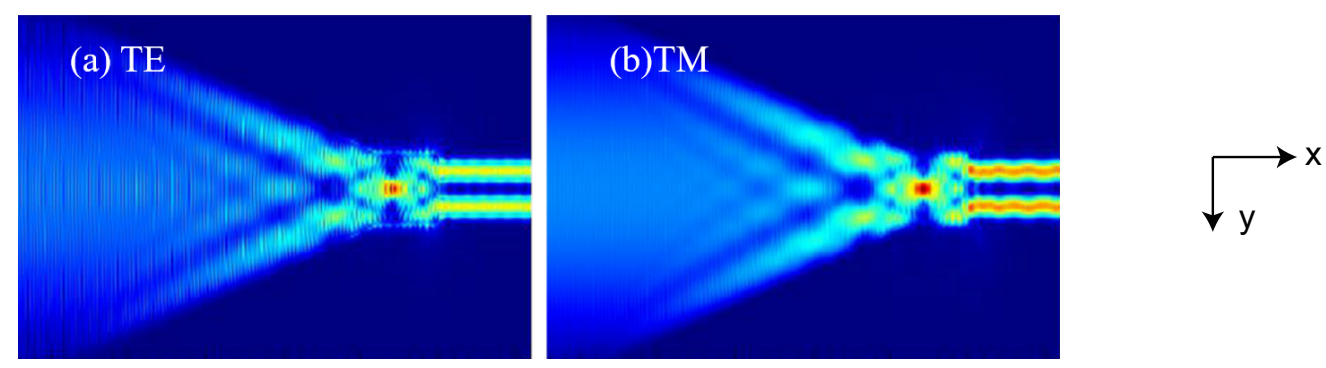

Figure 3. Energy distribution for both polarizations of the $1 \times 2$ beam splitter.

\subsection{The Output Taper Design}

Similarly to the analysis of $1 \times 2$ beam splitter for the input port, the output mode converter is a $1 \times 1 \mathrm{MMI}$ coupler, which uses the inverted taper instead of the single-mode waveguide as the output port. In order to realize the polarization-independent structure, the width of the multimode waveguide remains $2 \mu \mathrm{m}$. The simulated transmission results with different multimode waveguide lengths and wavelengths are shown in Figure 4.
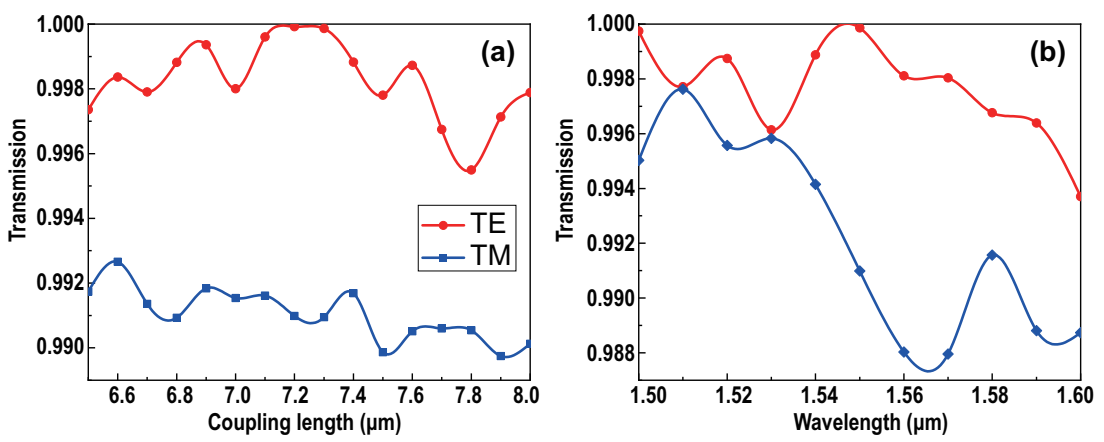

Figure 4. The transmission of the output taper versus (a) MMI length and (b) wavelength. TE polarization: red circle; TM polarization: blue diamond.

Figure 4a shows the transmission versus the length of the multimode waveguide of the output taper with a width of $2 \mu \mathrm{m}$ at the wavelength of $1.55 \mu \mathrm{m}$. At the length of $6.5 \mu \mathrm{m}$ to $8 \mu \mathrm{m}$, the output taper enables the high transmission of more than $99 \%$ for TE polarization. In particular, a highest transmission of $99.98 \%$ for TE polarization is achieved with the transmission of $99.1 \%$ for TM polarization at the length of $7.2 \mu \mathrm{m}$. The polarization-dependent loss is only $0.03 \mathrm{~dB}$. With the $7.2 \mu \mathrm{m}$ optimized length of the multimode waveguide of the output taper, the total length of the proposed output taper is $27.2 \mu \mathrm{m}$, which is one order of magnitude smaller than the normal waveguide taper with a length of more than $400 \mu \mathrm{m}$ [18].

Considering the operating wavelength bandwidth of the proposed $1 \times 2$ beam splitter, the transmission of the output taper from the wavelength from $1.5 \mu \mathrm{m}$ to $1.6 \mu \mathrm{m}$ is shown in Figure $4 \mathrm{~b}$. At the wavelength range of $100 \mathrm{~nm}$, the output taper displays great performance. The highest transmissions for TE and TM polarization are $99.98 \%$ and $99.76 \%$ at wavelengths of $1.55 \mu \mathrm{m}$ and $1.51 \mu \mathrm{m}$, respectively. Meanwhile, for the whole wavelength ranging from $1.5 \mu \mathrm{m}$ to $1.6 \mu \mathrm{m}$, PDLs are always below $0.04 \mathrm{~dB}$ and the transmissions are more than $98 \%$.

The output taper can also act as an input taper in the chip-based systems, which can convert the fiber's size field to the single-mode waveguide field. When two mirror symmetrical output tapers are oppositely connected together, one acts as the input taper, and the other acts as the output taper. The signal loading and downloading can be completed with optical fibers. Figure 5 shows the energy distribution for both polarizations at the center wavelength of $1.55 \mu \mathrm{m}$. The transmissions of $94.4 \%$ and $95 \%$ for TE and TM polarization are achieved, respectively. The total length is less than $60 \mu \mathrm{m}$, which is less than the normal signal loading and downloading systems with lengths longer than 
$800 \mu \mathrm{m}$ [24]. Additionally, the high performance of such structure enables the accurate testing of the photonic devices.
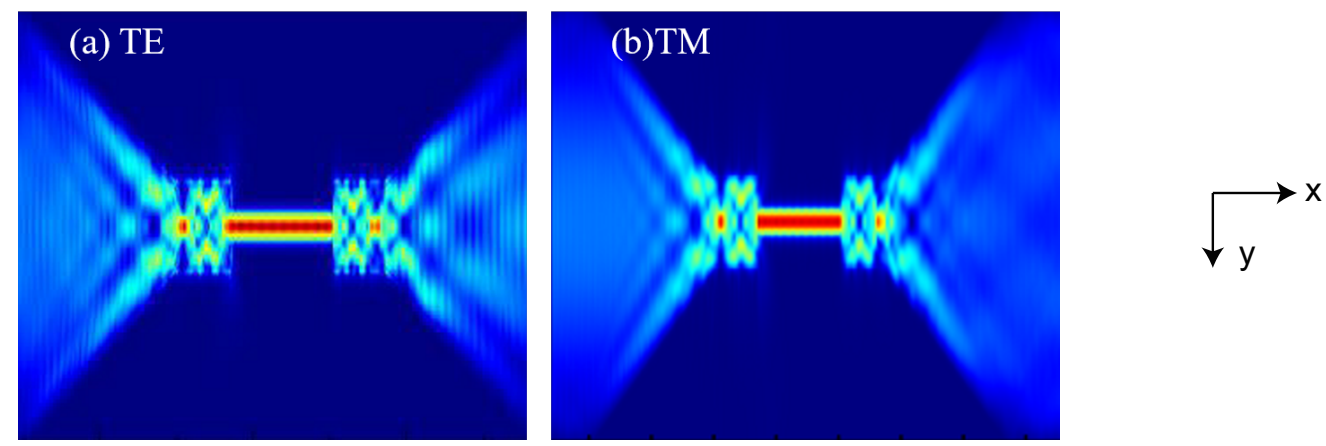

Figure 5. Energy distribution for both TE and TM polarizations with two output tapers being joined in opposite directions.

\subsection{The Performance of MZI}

If an optical field is present in the input waveguide taper of the $1 \times 2$ beam splitter, it will be separated into two single-mode waveguides, one of which has an additional phase shift by the phase shifter. Due to the interference, for different phase shifts, we obtain different portion of field at the two output single-mode waveguides of the MMI. With the different polarization of the incident optical field and different phase shifts, the corresponding optical fields in two output tapers are shown in Figure 6. When the TM/TE polarization field is input, with the phase shift of $\pi / 2$, the field will mostly come out at Channel 1, as shown in Figure $6 a, b$, while with the $3 \pi / 2$ phase shift, the field will mostly come out at Channel 2, as shown in Figure 6c,d. Here, we calculate the extinction ratio at the phase shift of $\pi / 2$ by $E R_{T E / T M}=-10 \log \frac{P_{C h 1, T E / T M}}{P_{C h 2, T E / T M}}$ [32], where $P_{C h 1, T E / T M}$ is the transmitted power of Channel 1 with TE or TM polarization and $P_{C h 2, T E / T M}$ is the transmitted power of Channel 2 with TE or TM polarization. Figure 7 shows the extinction ratio for TE and TM polarizations when the wavelength range varies from $1.5 \mu \mathrm{m}$ to $1.6 \mu \mathrm{m}$. The calculated ER of our design is $>18 \mathrm{~dB}$ in the $100 \mathrm{~nm}$ wavelength range for TE and TM polarizations. Then, we investigate the visibility of the proposed MZI structure. By tuning the phase shift of the phase shifter, the output power at Channel 1 and Channel 2 shows the interference fringes. The interference visibility is described as $V=\frac{P_{\max }-P_{\min }}{P_{\max }+P_{\min }}$, where $P_{\max }$ and $P_{\min }$ are the maximum and minimum output power of the interference fringe. For Channel 1, we find a visibility of $99.2 \%$ and $99.02 \%$ for TE and TM polarization at $1550 \mathrm{~nm}$, respectively.
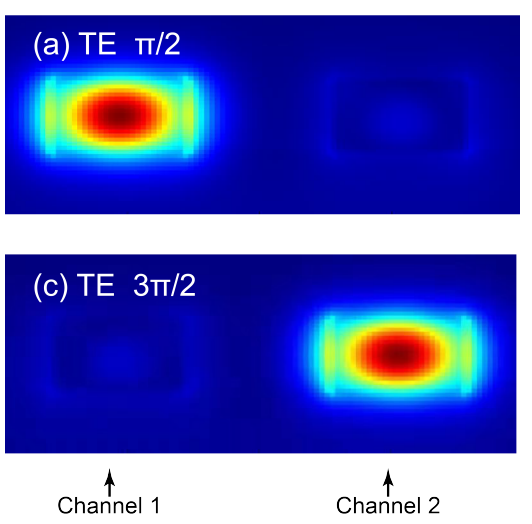
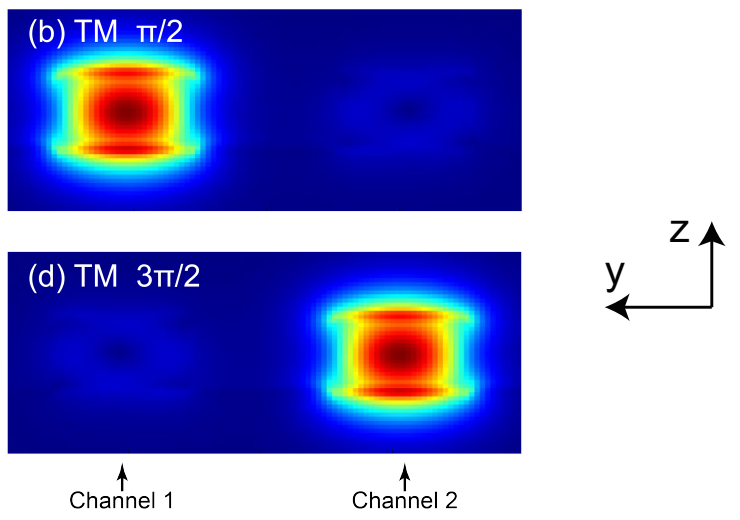

Figure 6. The output mode profiles for TE and TM polarizations at Channel 1 and Channel 2 with respect to different phase shifts at $1550 \mathrm{~nm}$. (a,c) Output mode profiles of TE polarization with $\pi / 2$ and $3 \pi / 2$ phase shift. (b,d) Output mode profiles of TM polarization with $\pi / 2$ and $3 \pi / 2$ phase shift. 


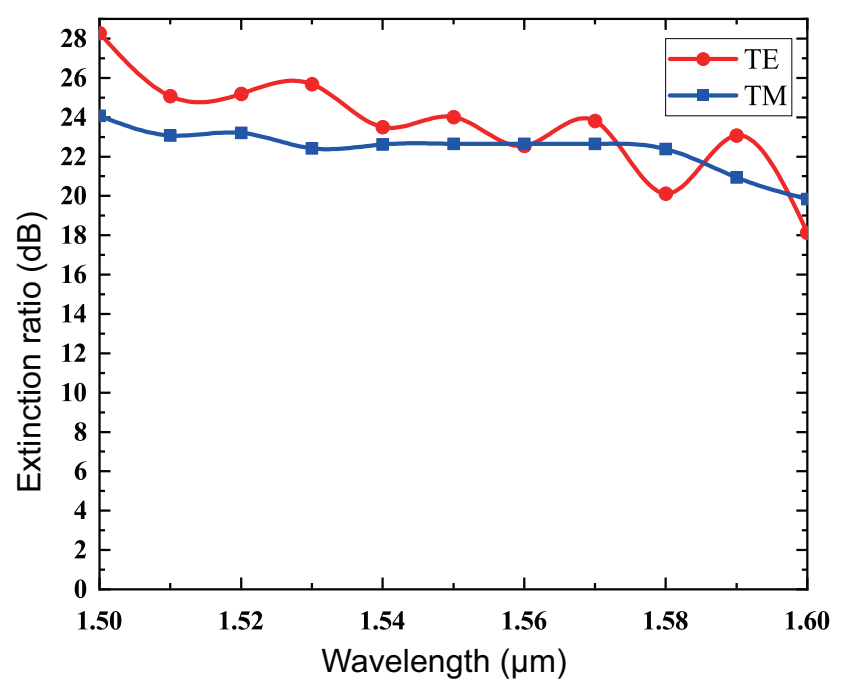

Figure 7. The simulated extinction ratio of the MZI for both TE and TM polarizations.

\section{Conclusions}

In summary, based on standard SOI platforms, we proposed a Mach-Zehnder switch that held the extinction ratio higher than $18 \mathrm{~dB}$ with large fabricated tolerance, low crosstalk, and wide operating wavelength bandwidth. By carefully designing the geometric dimension of the $1 \times 2$ polarization insensitive beam splitter whose input port is an input multimode waveguide taper and outputs are two single mode waveguides based on selfimage principle, the size of signal loading/downloading structure for the on-chip systems are reduced by one order of magnitude. In the operating wavelength bandwidth of $100 \mathrm{~nm}$, the total transmissions of the input structure and output structures are more than $95 \%$ for both TE and TM polarizaiton with an ultrashort length of $51.3 \mu \mathrm{m}$. Due to the complex mode in optical fiber, the polarization dependence of grating coupling device causes signal loss. Although polarization-maintaining fiber can solve the problem of mode mixing in optical fiber, its high cost and instability are not suitable for a large-scale integrated communication system. Therefore, studying the polarization-insensitive waveguide device is an effective way to solve the above problems. All components of the proposed structure hold great potential applications in the compact integration of the optical integrated circuit. The advantages of polarization insensitivity and the high interference contrast of this work provide an important strategy regarding research of monolithic integrated photonics in sensing, detection, optical computer, and quantum interconnection networks.

Author Contributions: Conceptualization and supervision, J.Z.; writing draft and preparation, H.C.; project administration, J.Y.; drawing work of this paper, X.S., C.M. and H.Z.; formal analysis, Z.Z.; investigation, W.Z., L.L. and Y.Y.; revision: X.C. All authors have read and agreed to the published version of the manuscript.

Funding: This work was supported by the National Natural Science Foundation of China (60907003, 61805278, 62005107, 62005074); the Foundation of NUDT (JC13-02-13, ZK17-03-01); the Hunan Provincial Natural Science Foundation of China (13JJ3001); the Program for New Century Excellent Talents in University (NCET-12-0142); the Natural Science Research Project of Higher Education Institutions of Jiangsu Province (20KJB140007); Natural Science Foundation of Jiangsu Province (BK20180862, BK20190839); China Postdoctoral Fund (2019M651725).

Institutional Review Board Statement: Not applicable.

Informed Consent Statement: Not applicable.

Data Availability Statement: The data that support the findings of this study are available from the corresponding author upon reasonable request.

Conflicts of Interest: The authors declare no conflict of interest. 


\section{References}

1. Politi, A.; Cryan, M.J.; Rarity, J.G.; Yu, S.; O’Brien, J.L. Silica-on-Silicon Waveguide Quantum Circuits. Science 2008, 320, 646-649. [CrossRef]

2. Matthews, J.; Politi, A.; Stefanov, A.; O’Brien, J.L. Manipulating multi-photon entanglement in waveguide quantum circuits. Nat. Photonics 2009, 3, 346-350. [CrossRef]

3. Bindal, P.; Sharma, A. Modeling of Ti:LiNbO/sub 3/waveguide directional couplers. IEEE Photonics Technol. Lett. 1992, 4, 728-731. [CrossRef]

4. Gregory, I.M. Design and Stability Analysis of an Integrated Controller for Highly Flexible Advanced Aircraft Utilizing the Novel Nonlinear Dynamic Inversion. Ph.D. Thesis, California Institute of Technology, Pasadena, CA, USA, 2004.

5. Zhang, M.; Chen, K.; Jin, W.; Chiang, K.S. Electro-optic mode switch based on lithium-niobate Mach-Zehnder interferometer. Appl. Opt. 2016, 55, 4418-4422. [CrossRef] [PubMed]

6. Wang, H.; Li, X.; Zhang, M.; Chen, K. Broadband $2 \times 2$ lithium niobate electro-optic switch based on a Mach-Zehnder interferometer with counter-tapered directional couplers. Appl. Opt. 2017, 56, 8164-8168. [CrossRef] [PubMed]

7. Barth, C.; Wolters, J.; Schell, A.W.; Probst, J.; Schoengen, M.; Löchel, B.; Kowarik, S.; Benson, O. Miniaturized Bragg-grating couplers for SiN-photonic crystal slabs. Opt. Express 2015, 23, 9803-9811. [CrossRef]

8. Lu, L.; Xia, L.; Chen, Z.; Chen, L.; Ma, X.S. Three-dimensional entanglement on a silicon chip. NPJ Quantum Inf. 2020, 6, 1-9. [CrossRef]

9. Peruzzo, A.; Lobino, M.; Matthews, J.C.F.; Matsuda, N.; Politi, A.; Poulios, K.; Zhou, X.Q.; Lahini, Y.; Ismail, N.; Wörhoff, K.; et al. Quantum Walks of Correlated Photons. Science 2010, 329, 1500-1503. [CrossRef] [PubMed]

10. Tao, S.H.; Fang, Q.; Song, J.F.; Yu, M.B.; Lo, G.Q.; Kwong, D.L. Cascade wide-angle Y-junction $1 \times 16$ optical power splitter based on silicon wire waveguides on silicon-on-insulator. Opt. Express 2008, 16, 21456-21461. [CrossRef]

11. Yamada, H.; Tao, C.; Ishida, S.; Arakawa, Y. Optical directional coupler based on Si-wire waveguides. IEEE Photonics Technol. Lett. 2005, 17, 585-587. [CrossRef]

12. Suzuki, K.; Cong, G.; Tanizawa, K.; Kim, S.H.; Ikeda, K.; Namiki, S.; Kawashima, H. Ultra-high-extinction-ratio $2 \times 2$ silicon optical switch with variable splitter. Opt. Express 2015, 23, 9086-9092. [CrossRef] [PubMed]

13. Bonneau, D.; Engin, E.; Ohira, K.; Suzuki, N.; Yoshida, H.; Iizuka, N.; Ezaki, M.; Natarajan, C.M.; Tanner, M.G.; Hadfield, R.H.; et al. Quantum interference and manipulation of entanglement in silicon wire waveguide quantum circuits. New J. Phys. 2012, 14, 045003. [CrossRef]

14. Wang, J.; Paesani, S.; Ding, Y.; Santagati, R.; Skrzypczyk, P.; Salavrakos, A.; Tura, J.; Augusiak, R.; Mančinska, L.; Bacco, D.; et al. Multidimensional quantum entanglement with large-scale integrated optics. Science 2018, 360, 285-291. [CrossRef] [PubMed]

15. Qiang, X.; Zhou, X.; Wang, J.; Wilkes, C.M.; Loke, T.; O'Gara, S.; Kling, L.; Marshall, G.D.; Santagati, R.; Ralph, T.C.; et al. Large-scale silicon quantum photonics implementing arbitrary two-qubit processing. Nat. Photonics 2018, 12, 534-539. [CrossRef]

16. Ding, Y.; Bacco, D.; Dalgaard, K.; Cai, X.; Zhou, X.; Rottwitt, K.; Oxenløwe, L.K. High-dimensional quantum key distribution based on multicore fiber using silicon photonic integrated circuits. NPJ Quantum Inf. 2017, 3, 25. [CrossRef]

17. Wilkes, C.M.; Qiang, X.; Wang, J.; Santagati, R.; Paesani, S.; Zhou, X.; Miller, D.; Marshall, G.D.; Thompson, M.G.; O’Brien, J.L. 60dB high-extinction auto-configured Mach-Zehnder interferometer. Opt. Lett. 2016, 41, 5318-5321. [CrossRef]

18. Cheng, Z.; Tsang, H.K. Experimental demonstration of polarization-insensitive air-cladding grating couplers for silicon-oninsulator waveguides. Opt. Lett. 2014, 39, 2206-2209. [CrossRef]

19. Xu, Y.; Xiao, J. An Ultracompact Polarization-Insensitive Silicon-Based Strip-to-Slot Power Splitter. IEEE Photonics Technol. Lett. 2016, 28, 536-539. [CrossRef]

20. Zhang, J.; Yang, J.; Liang, L.; Wu, W. Broadband TM-mode-pass polarizer and polarization beam splitter using asymmetrical directional couplers based on silicon subwavelength grating. Opt. Commun. 2018, 407, 46-50. [CrossRef]

21. Wang, J.; Bonneau, D.; Villa, M.; Silverstone, J.W.; Santagati, R.; Miki, S.; Yamashita, T.; Fujiwara, M.; Sasaki, M.; Terai, H.; et al. Chip-to-chip quantum photonic interconnect by path-polarization interconversion. Optica 2016, 3, 407-413. [CrossRef]

22. Bennett, C.; Brassard, G. Quantum Cryptography: Public Key Distribution and Coin Tossing. In Proceedings of the IEEE International Conference on Computers, Systems, and Signal Processing, Bangalore, India, 9-12 December 1984; pp. 175-179.

23. Wang, S.; Dai, D. Polarization-insensitive $2 \times 2$ thermo-optic Mach-Zehnder switch on silicon. Opt. Lett. 2018, 43, 2531-2534. [CrossRef]

24. Zhang, J.; Yang, J.; He, X.; Jie, H.; Zhang, Z. Ultrashort and efficient adiabatic waveguide taper based on thin flat focusing lenses. Opt. Express 2017, 25, 19894-19903. [CrossRef] [PubMed]

25. Liu, Y.; Sun, W.; Xie, H.; Zhang, N.; Xu, K.; Yao, Y.; Xiao, S.; Song, Q. Adiabatic and Ultracompact Waveguide Tapers Based on Digital Metamaterials. IEEE J. Sel. Top. Quantum Electron. 2019, 25, 1-6. [CrossRef]

26. Sethi, P.; Haldar, A.; Selvaraja, S.K. Ultra-compact low-loss broadband waveguide taper in silicon-on-insulator. Opt. Express 2017, 25, 10196-10203. [CrossRef]

27. Zhang, J.; Kai, G.; Gao, M.; Yang, G.; Yang, J. Design of polarization-insensitive high-visibility silicon-on-insulator quantum interferometer. Sci. Rep. 2018, 8, 1-7. [CrossRef] [PubMed]

28. Shah, M.K.; Lu, R.; Peng, D.; Ma, Y.; Ye, S.; Zhang, Y.; Zhang, Z.; Liu, Y. Graphene-Assisted Polarization-Insensitive Electroabsorption Optical Modulator. IEEE Trans. Nanotechnol. 2017, 16, 1004-1010. [CrossRef] 
29. Liu, M.; Yin, X.; Ulin-Avila, E.; Geng, B.; Zentgraf, T.; Ju, L.; Wang, F.; Zhang, X. A graphene-based broadband optical modulator. Nature 2011, 474, 64-67. [CrossRef]

30. Soldano, L.B.; Pennings, E.C.M. Optical multi-mode interference devices based on self-imaging: Principles and applications. J. Light. Technol. 1995, 13, 615-627. [CrossRef]

31. Lumerical FDTD. Available online: https://www.lumerical.com/tcad-products/fdtd/ (accessed on 12 September 2021). [CrossRef]

32. Dai, D.; Wang, Z.; Peters, J.; Bowers, J.E. Compact Polarization Beam Splitter Using an Asymmetrical Mach-Zehnder Interferometer Based on Silicon-on-Insulator Waveguides. IEEE Photonics Technol. Lett. 2012, 24, 673-675. 